By James Goodwin and Sarah Lock 


\section{Bridging the Gap - How the Global Council on Brain Health Delivers to the People of the World What Science Is Telling Us about Brain Aging}

By James Goodwin and Sarah Lock

W orldwide, the number of people with dementia increased from 25 million to nearly 35 million between 2005 and 2010, and this figure is projected to increase to more than 75 million by 2030 and 135 million by 2050 . Most people with dementia live in low- and middle-income countries, and the proportion is projected to rise from 62 percent in 2013 to 71 percent by 2050 . Increases will therefore be even sharper in developing countries, and the growth of dementia cases in Latin America is predicted to exceed that in any other region of the world. ${ }^{1}$

The estimated annual cost worldwide of dementia is USD 604 billion, or 1 percent of global gross domestic product (GDP). ${ }^{2}$ In high-income countries, informal care (i.e., unpaid care provided by the family; 45 percent) and formal social care (40 percent) account for most of the costs, while the proportionate contribution of direct medical costs (15 percent) is much lower. In low- and middle-income countries, direct social care costs are small, and informal care costs predominate; however, in the coming decades, changing demography in many low- and middle-income countries may lead to a decline in the availability of extended family members to provide care.

Yet dementia is just the tip of the iceberg. Though a recent analysis by Alzheimer's Society ${ }^{3}$ suggests that 7.1 percent of people ages 65 and over have dementia in the United Kingdom, it is estimated that, at any one time, between 15 percent and 20 percent of people 65 and older people have some form of mild cognitive impairment. ${ }^{4}$ It is estimated that before the onset of dementia, between 20 to 30 years of neurodegeneration in the brain has already occurred. Many older adults also worry about normal age-related changes to the brain that result in declines to mental functioning such as attention, thinking, memory, and decision making. AARP surveys have consistently showed that staying mentally sharp is a top concern of 87 percent of aging adults in the United States.

In spite of the many thousands of research papers, publications, books, and media reports about dementia and cognitive decline, there is still wide misunderstanding among the general public about brain health and aging. Agerelated decline is greatly feared, largely because many regard age-related losses as inevitable - and because these individuals are concerned that any change in brain function means they are likely to have dementia. Advancing age increases the risk for other health conditions, such as heart disease and diabetes. For these diseases, the communication of scientific and medical advances has been much more successful - heart disease, diabetes, and even cancer are now considered manageable conditions that people can live with; dementia is often thought of as an immediate death sentence.

In the wake of the G8 Dementia Summit in London in 2013, the world has focused its attention on dementia - its diagnosis, cure, and long-term care. There has been comparatively little attention paid to dementia's invisible onset and how to reduce risks factors or cognitive decline. Even though it has been well known for some time that there are 20 to 30 years of change in the brain across the life course - some of which is normal cognitive aging and some of which precedes a diagnosis of dementia - only recently have we begun to examine the public health aspects of reducing 

are high, they are exceeded by those who are incapacitated by mild cognitive impairment or cognitive aging, and dwarfed by those individuals who are worried they soon may be facing such conditions.”

those risks. ${ }^{5}$ Though the numbers of adults with dementia are high, they are exceeded by those who are incapacitated by mild cognitive impairment or cognitive aging, and dwarfed by those individuals who are worried they soon may be facing such conditions.

It is not surprising that, along with the rise in the age of our world's population, interest and concern about keeping all those aging brains functioning as well as they can across growing lifespans has likewise been on the rise. Two years ago, to address these vital concerns, AARP and Age UK launched the Global Council on Brain Health (GCBH), an international collaboration among scientists, health professionals, scholars, and policy experts, to address how to help people maintain their abilities to think and reason as they age, and the developing science in this area.

To date, GCBH has convened five meetings of expert panels, which have considered important risk factors for brain health. These areas are physical activity, sleep, social engagement, brain-stimulating activity, and nutrition. These panels have been convened in Toronto; Washington, DC; Vancouver;
London; and Baltimore. To make them truly representative of cultures worldwide, GCBH has a "four continents rule" so that each expert panel consists of issue specialists from around the world. Nineteen countries involved so far have included Australia, South Africa, Argentina, Israel, China, Japan, Singapore, Brazil, many in Europe, Canada, and the United States. These expert, international panels review the evidence and produce, first, a "consensus statement" that summarizes what can be said about the current state of science about each factor and, second, provides guidelines or recommendations for public consumption. The reports of these panels, have all been published widely and infographics created from the GCBH's recommendations have been translated into several languages, including Spanish, Chinese, Arabic, and French.

The reports have attracted immense interest globally from the media and from consumers. For example, after a sleep report was published, Age UK was interviewed on BBC national radio and 31 local radio stations, on BBC television, on Sky News, and in the major printed media in London. The

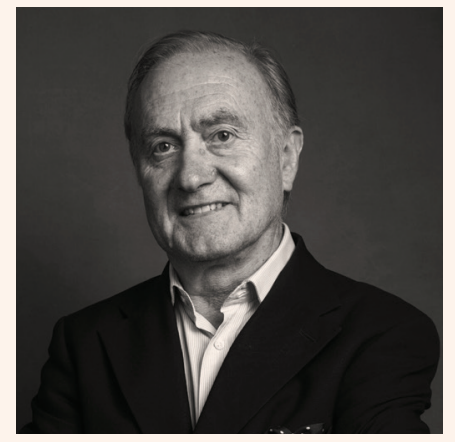

James Goodwin, PhD CHIEF SCIENTIST AGE UK

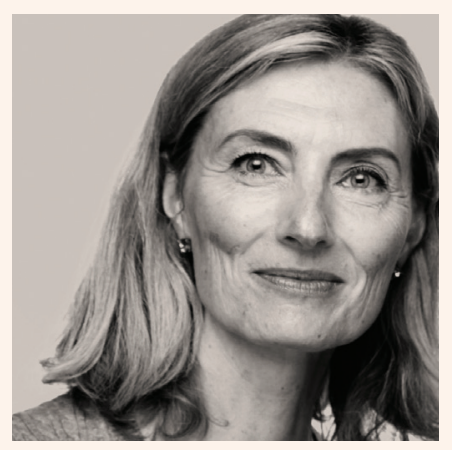

Sarah Lock, JD

SENIOR VICE PRESIDENT POLICY, AARP 


\section{Debunking Myths About the Aging Brain}

7 Facts About Your Brain

MYTH

You are born with all the neurons that your brain will ever have.
FACT

Neurons are continually created throughout your life in areas of the brain through a process called neurogenesis.
We don't really know how the brain works.
Learning can happen at any age when you get involved with cognitively stimulating activities like meeting new people or trying new hobbies.
You can't learn new things when you are old.
Researchers have made great strides in understanding the brain in recent years. The field of neuroscience is at the cusp of new and exciting breakthroughs.

\section{Dementia is an inevitable consequence of old age.}

Dementia is not a normal part of aging. There is a big difference between typical agerelated changes in the brain and those that are caused by disease.

\section{Only young people can learn \\ a new language.}

While it may seem simpler for kids - with simpler sentence structures and a lack of self-consciousness - your age isn't a limitation in learning a new language.

\footnotetext{
Older people are doomed to forget things.
}

Remembering details is easier for some people than others, but this is true of all ages. There are strategies to help remember names, facts, etc. and paying closer attention can often help you remember better.

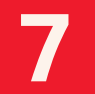

A person who has memory training never forgets.
Keep practicing your memory skills. "Use it or lose it" applies to memory training in the same way it applies to maintaining your physical health. 
“...some of the best ways to support and maintain your memory, reasoning skills, and ability to focus are to engage in formal or informal educational activities, learn a new language, engage in work or leisure
activities that are mentally challenging,
and connect socially with others."

most recent press release on cognitively stimulating activities garnered more than 34 million media impressions in the United States and international coverage in India, Argentina, Australia, and Great Britain. Recently in San Francisco, GCBH delivered a symposium on its work to a packed meeting of the International Association of Gerontology and Geriatrics (IAGG) 2017 World Congress.

GCBH's latest report, Engage Your Brain: GCBH Recommendations on Cognitively Stimulating Activities, available at www.GlobalCouncilonBrainHealth.org, challenges two widely held outdated beliefs about aging and the brain - first, that cognitive decline is inevitable as you age, and second, that brain games are the best way to keep your brain sharp as you age. In fact, the report convincingly shows that people can help keep their brain sharp and that they should not let age limit them.

By continually finding new ways to challenge the way you think, and by choosing to combine mentally engaging activities along with physical exercise, it is indeed possible to change your brain at any age. As to the second common misconception - that brain games are the best way to keep your brain agile over time - the $\mathrm{GCBH}$ found that the evidence for the long-term brain health benefits of what most people consider "brain games" is weak to nonexistent. Cognitive training is a global multibillion-dollar industry and has recently been getting a great deal of attention in the print and electronic media. It's no wonder. The appeal of having fun while playing games and doing something good for your brain may be more attractive to the couchpotato instincts in all of us. Yet, there are many mentally engaging activities that can help your brain stay sharp over your lifespan and you don't have to pay for expensive brain-training games in order to reduce your risk for cognitive decline. GCBH says that some of the best ways to support and maintain your memory, reasoning skills, and ability to focus are to engage in formal or informal educational activities, learn a new language, engage in work or leisure activities that are mentally challenging, and connect socially with others. As for physical exercise, while it takes work to keep up physical activity, and sometimes engaging with other people can be exhausting, it is worth the sustained attention and effort because, as GCBH points out, scientific evidence confirms that these efforts can pay off in maintaining brain health.

$\mathrm{GCBH}$ recognizes that much is yet to be done, and there are many more areas to explore. The GCBH's nutrition report on how diet can impact brain health is expected to be released in early 2018. Future topics will include mental well-being, dietary supplements, and sensory health (e.g., age-related hearing and sight impairments). But our vision and hope is that - similar to our knowledge about cardiovascular health
— there will eventually be widespread and sound public understanding that we ourselves can do much to maintain our brain health regardless of culture, country, or community. •

\footnotetext{
${ }^{1}$ Sujata Ray and Susan Davidson PhD (2014). Dementia and cognitive decline: a review of the evidence. London, Age UK. https://www.ageuk.org.uk/globalassets/age-uk/documents/ reports-and-publications/reports-and-briefings/health--wellbeing/rb_oct14_cognitive_decline_and_dementia_evidence _ review_age_uk.pdf

${ }^{2}$ Alzheimer's Disease International, World Alzheimer Report 2010 ([[London:]] Alzheimer's Disease International, 2010).

${ }^{3}$ Alzheimer's Society, Dementia UK, 2nd ed. ([London:]] Alzheimer's Society, 2014), http://alzheimers.org.uk/site/scripts/ download_info.php?fileID=2323.

4 Alzheimer's Society, "Factsheet: Mild Cognitive Impairment," Alzheimer's Society, [London,]] 2014, http://www.alzheimers. org.uk/site/scripts/download_info.php?fileID=1773.

${ }^{5}$ Institute of Medicine, Cognitive Aging: Progress in Understanding and Opportunities for Action (Washington, DC: The National Academies Press, 2015).
} 
AARP International engages global stakeholders to spark solutions that strengthen communities, protect the vulnerable and enable people around the world to pursue their goals and dreams. Working with governments, civil society and the private sector, we are focused on enhancing the quality of life for people as they age. We serve as the global voice for AARP, a social change organization with a membership of more than 37 million.

THE JOURNAL ONLINE

aarpinternational.org/journal

AARP INTERNATIONAL ONLINE

aarpinternational.org

FACEBOOK

facebook.com/aarpintl

TWITTER

@aarpintl

CONTACT

AARP International

601 E Street, NW

Washington, DC 20049

United States

T +1 2024342398

E international@aarp.org

AARP International: The Journal is a publication of AARP. The views expressed herein do not necessarily represent policies of AARP and should not be construed as endorsements. The mention of a product or service herein is solely for information to our readers and may not be used for any commercial purpose. AARP, which was established in 1958, is a nonprofit, nonpartisan organization with tens of millions of members ages 50 and older. State offices are located in all 50 states, the District of Columbia, Puerto Rico, and the Virgin Islands.

January 2018

()2018, AARP

Reprinting with permission only. 